\title{
THE CONTROL STRATEGY FOR DEGREES OF FREEDOM IN REMOTE PREHENSION WITH A TOOL
}

\author{
Bin Zheng ${ }^{1}$, Christine L. MacKenzie ${ }^{2}$ \\ ${ }^{1}$ Legacy Health System, Portland, Oregon ${ }^{2}$ Simon Fraser University, Burnaby, British Columbia, Canada \\ Constructing movement couplings is essential for decreasing degrees-of-freedom for a compound \\ movement that requires coordination over a multiple segments. Angular movements of joints in the upper \\ limbs are examined, the pattern of movement couplings between prehension performed with the hands \\ (natural prehension) and with a simple grasper held in the hands (remote prehension). In remote \\ prehension, the shoulder and elbow joint are tightly associated with a clear in-phase joint to joint \\ movement; the elbow and wrist display both anti- and in-phase movements due to the change of initial \\ configuration of the upper limb when holding a tool. In contrast, the shoulder-elbow bond is mixed in \\ natural prehension, but the elbow and wrist bond is predominant with an anti-phase pattern. With diversity \\ for joint couplings, the movement consistency of the hinge is preserved with relatively smaller path \\ variability. Results support the end-point control notion, i.e. movement is controlled by extrinsic \\ coordinates close to the end-effectors of the movement system.
}

\section{INTRODUCTION}

Degrees of freedom (DOF) are defined as the number of independent coordinates required for determining a system position. The problem of DOF in human movement control was first described by Nicolas Bernstein as fellows: for any given movement, the total resource of degrees of freedom from which a coordinated activity can be assembled exceeds the number of degrees of freedom that are minimally necessary to accomplish that task (Bernstein, 1967; Whiting \& Bernstein, 1984). When a tool is used, extra DOF are introduced into the motor control system. To build movement coordination with a tool, the exceeded number of DOF must be reduced to a level where the function of the tool is fulfilled without loading superfluous mental or physical stresses to the user.

Bernstein postulated possible solutions for controlling DOF and argued such strategies are directly associated with the motor skill of a human operator (Bernstein, 1967). According to Bernstein, a human operator tends to "freeze out" a portion of the DOF set in the early stage of skills learning. Some strong and rigid couplings are formed in this stage among multiple movement segments. As movement skills improve, the frozen movement segments will be released, and those rigid couplings between segments can turn into a dynamic, controllable, and energy-efficient synergic unit. The releasing process of couplings was subject to the context of the task and the anatomy structure of the body (Vereijken et al., 1992).

When a coupling is formed between two movement segments, movement parameters will display a certain level of coordination. Conventionally, angular movement of joints and the synchronization of the angle changes of the joints are used to measure the couplings of motor segments (Vereijken et al., 1992). Also, the path variability of a movement segment may reduce when it is frozen. Changes of joint angles during a movement is utilized in this study to catch the movement couplings during prehensions preformed by the hand directly (natural prehension) and holding a grasper in the hand (remote prehension).

In this study, angular movements of the shoulder, elbow, and wrist were computed and the angular changes were correlated between joints. We predicted that a tighter linkage among joints of the upper limb would be observed in remote prehension compared to natural prehension. This hypothesis was generated based on an assumption that human users would freeze some internal DOF of the limbs when extra DOF were introduced by using a tool; thus overall DOF would be manageable to accomplish a task goal. Certainly, the degree of such initial freezing on DOF would vary with the task requirement and tool mechanical properties.

To examine Bernstein's coupling notion of DOF control for different learning phases, two levels of motor skills were introduced by comparing task performance between the preferred and nonpreferred hand. The preferred hand is believed to have higher skill level than the non-preferred hand (Elliott et al., 1993; Roy \& Elliott, 1986). We predicted that the above difference between natural and remote prehension would be more pronounced when the task was performed by the nonpreferred hand than the non-preferred hand.

Two levels of task requirement were introduced in the study by changing the diameter of the base which supports the object. When the object was placed on the top of a narrow base, greater precision was required compared to grasping the same object placed on a wide base. A previous study has shown that path variability from the end-effector of the motor system was reduced when task precision requirement escalated (Bertram, 2002). In addition to the sensors (IREDs) placed on the chest, shoulder, elbow, wrist and thumb, an IRED was placed on the hinge of the tool in remote prehension. Variability of path trajectory was computed from above body and tool sites. 
We predicted that the path variability from the tools would be smaller from the tool than the wrist. The overall path variability would be reduced in remote compared to natural prehension. The above differences between remote and natural prehension would be more pronounced when reaching to object on the narrow base than the wide base. The overall goal of the study was to examine the strategy for DOF control in tool use.

\section{METHODS}

Twelve university students who were naive to the purpose of the study participated in the study. Ethical approval was obtained from the Simon Fraser University. The task was to reach, grasp, and lift up a dowel $(2 \mathrm{~cm}$ in diameter) from either a wide $(5 \mathrm{~cm})$ or a narrow $(1 \mathrm{~cm})$ base using the preferred and non-preferred hand with and without holding a simple grasper. When used, a grasper (pivot ratio $=1: 1$ ) was attached to the thumb and the index finger.

The 3D spatial paths of the IREDs of the chest, shoulder, elbow, wrist, thumb and the hinge of the tool (only for remote prehension) were plotted. For each trial, the joint angle value was calculated for the shoulder, elbow, and wrist over the course of the movement by linking three IREDs in the vicinity of the joint. Cross-correlations between the shoulder and elbow, the elbow and wrist, and the wrist and shoulder were performed on angular movement data. In addition, path trajectory data were time-flipped and path variability was calculated frame-by-frame for $300 \mathrm{~ms}$ prior to the contact of the object (Bertram, 2002).

To obtain the global estimate of path variability for each IRED in each condition, the volume of the ellipsoid defined by the standard deviations in $x, y$, and $z$ dimensions was computed. In mathematics, an ellipsoid is a three-dimensional object. All planar cross-sections of an ellipsoid are either ellipses or circles. The volume of an ellipsoid is defined by the formula of $v=(4 \pi / 3) a b c$, where $v$ denoted as volume of ellipsoid; $\pi=3.1415926 ; a, b$ and $c$ are the semi-axes of an ellipsoid in each of three dimensions. In this study we used the standard deviations in $x, y$, and $z$ axes to replace $a, b$, and $c$. The idea of using the area of an ellipse calculations to estimate the global variability of 2D movement trajectories was first introduced by Georgopoulos in 1981(Georgopoulos et al., 1981), and followed by Paulingnan et al (Paulignan et al., 1991).

\section{RESULTS}

Reaching with the grasper generally led to an increase of the mean angle range of shoulder and elbow angles, but a decrease in angle ranges of the wrist, compared to the data in natural prehension. This phenomenon was more pronounced for the nonpreferred than the preferred hand.

Cross-correlations between shoulder and elbow angles showed different patterns between natural and remote prehension. In natural prehension, a bimodal distribution was shown, with in-phase (the shoulder and elbow extended and flexed synchronously) and anti-phase (the elbow extended but shoulder flexed) joint couplings both present (Figure 1A, 1C). In contrast, in remote prehension, anti-phase couplings were predominated by angular movement between the shoulder and the elbow, i.e. the shoulder angle decreased while the elbow angle increased (Figure 1B, 1D).

Different coupling patterns between natural and remote prehension were also observed between joints of the elbow and wrist. In natural prehension, the wrist extended for most of trials as the elbow extended, therefore an in-phase unimodal distribution was presented (Figure 2A, 2C). In remote prehension, association between the wrist and elbow joint angles was diverse. Adding a tool changes the configuration of the wrist prior to movement start which caused the wrist to either extend or flex when the elbow was extending (Figure 2B, 2D).

While the couplings displayed various patterns among different joints of upper limbs, path variability from limb and tool sites was significantly smaller in remote prehension compared to using the hand directly (Figure 3 ).

Relatively small path variability for remote prehension was displayed throughout the later movement phase prior to the contact of the object. The path variability measured from the hinge position of the tool was even smaller than the wrist (Figure 4). A dramatic decrease was also observed in path variability at the thumb, wrist, and elbow as the effectors approached the object. In comparison, the variability of the chest and shoulder was small compared to other body sites and the volume changed little during the courses of movement (Figure 3).

Analyses of path variability revealed interactions between task and hand at $250 \mathrm{~ms}$ and $300 \mathrm{~ms}$ prior to the contact of an object; in natural prehension the nonpreferred hand displayed larger path variability than using of the preferred hand. This reflects a higher degree of movement consistency when using the preferred hand in natural prehension. However, in remote prehension the nonpreferred hand had smaller overall path variability than the preferred hand. In the same experimental setting of reaching and grasping using a tool, we found that participants moved much more slowly when using the non-preferred hands, and they had a longer deceleration phased compared to using the preferred hand (Zheng \& MacKenzie, 2007). These kinematic results suggest that the non-preferred hand was moved more cautiously with a tool than the preferred hand in order to achieve precise and consistent movements from trial to trial. 

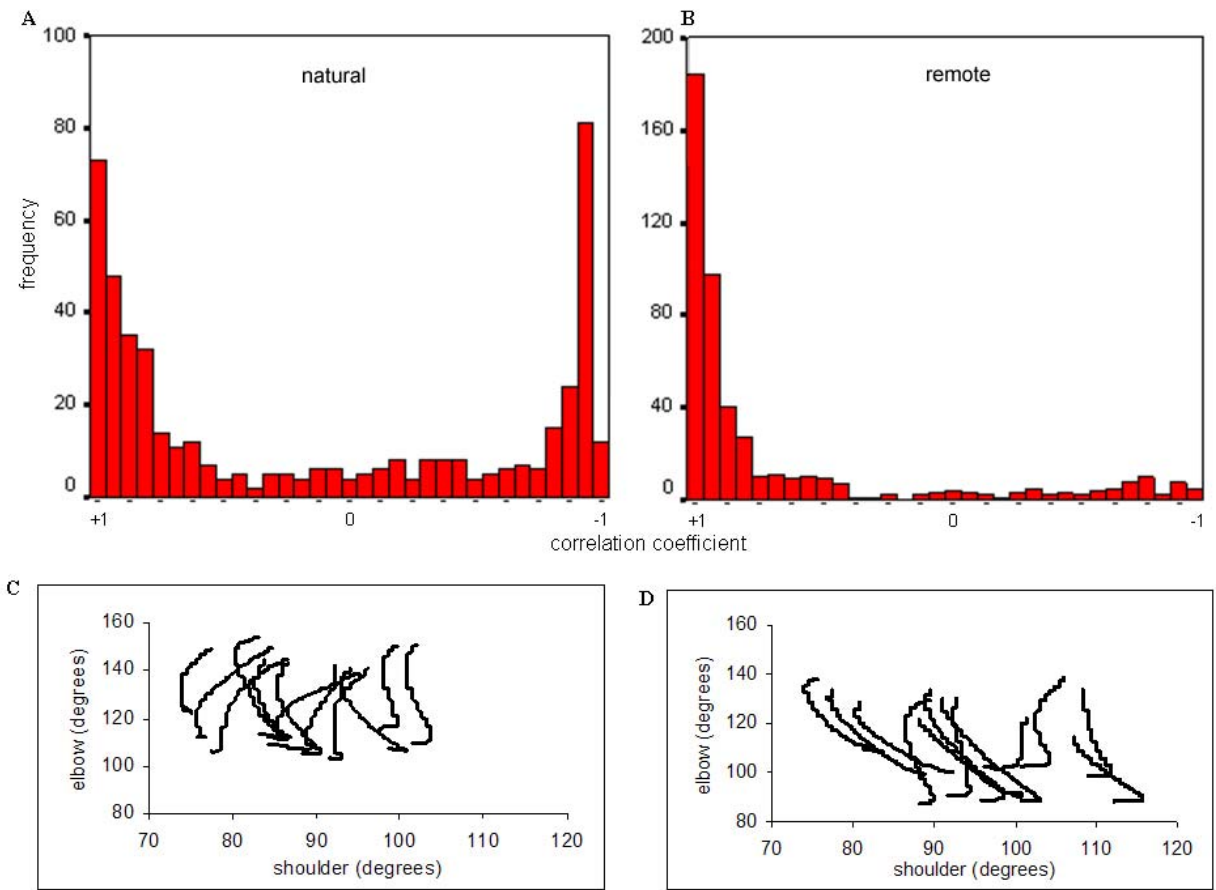

Figure 1. Correlation of angular movements between shoulder and elbow joint. Top row, distribution of correlation coefficients; bottom row, angle-angle plot. Left column: natural prehension; Right: remote prehension
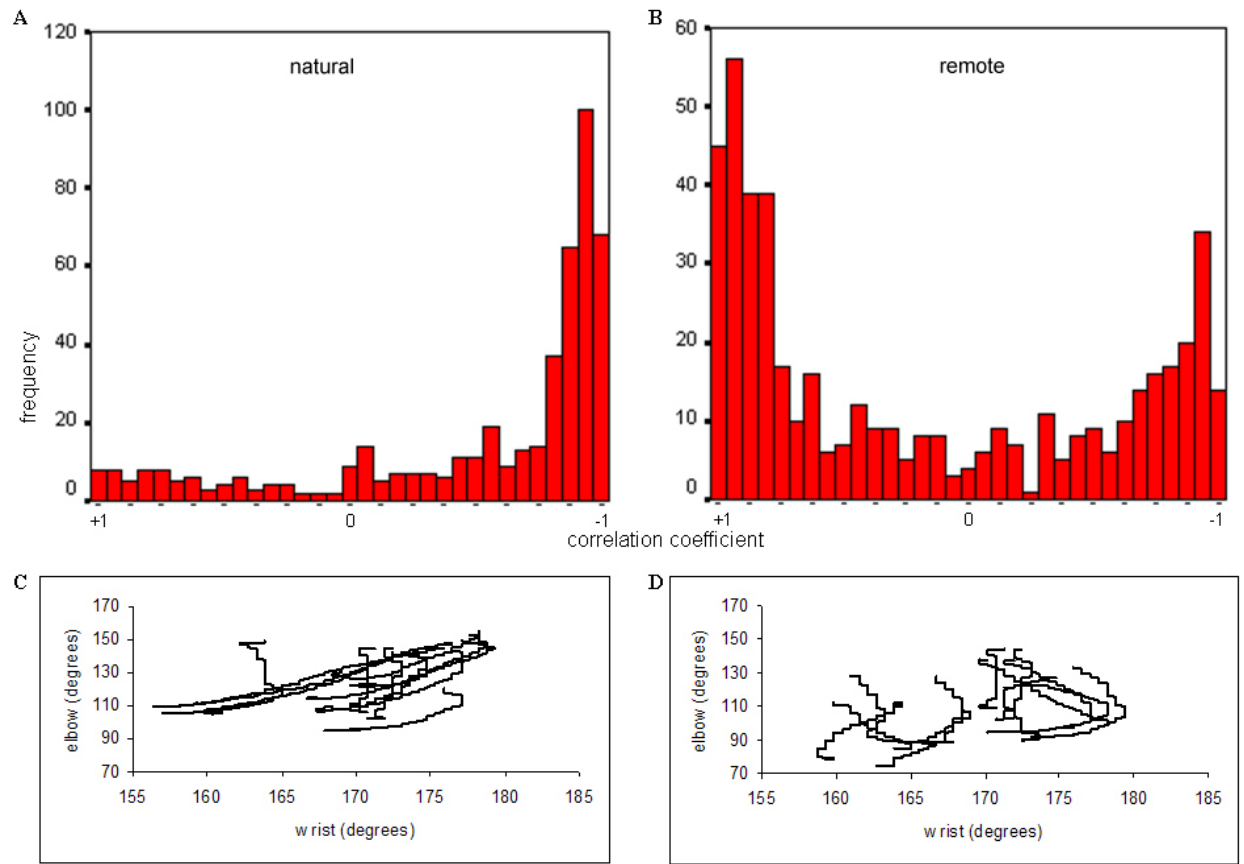

Figure 2. Correlation of angular movements between elbow and wrist joint. Top row, distribution of correlation coefficients; bottom row, angle-angle plot. Left column: natural prehension; Right: remote prehension 
hand
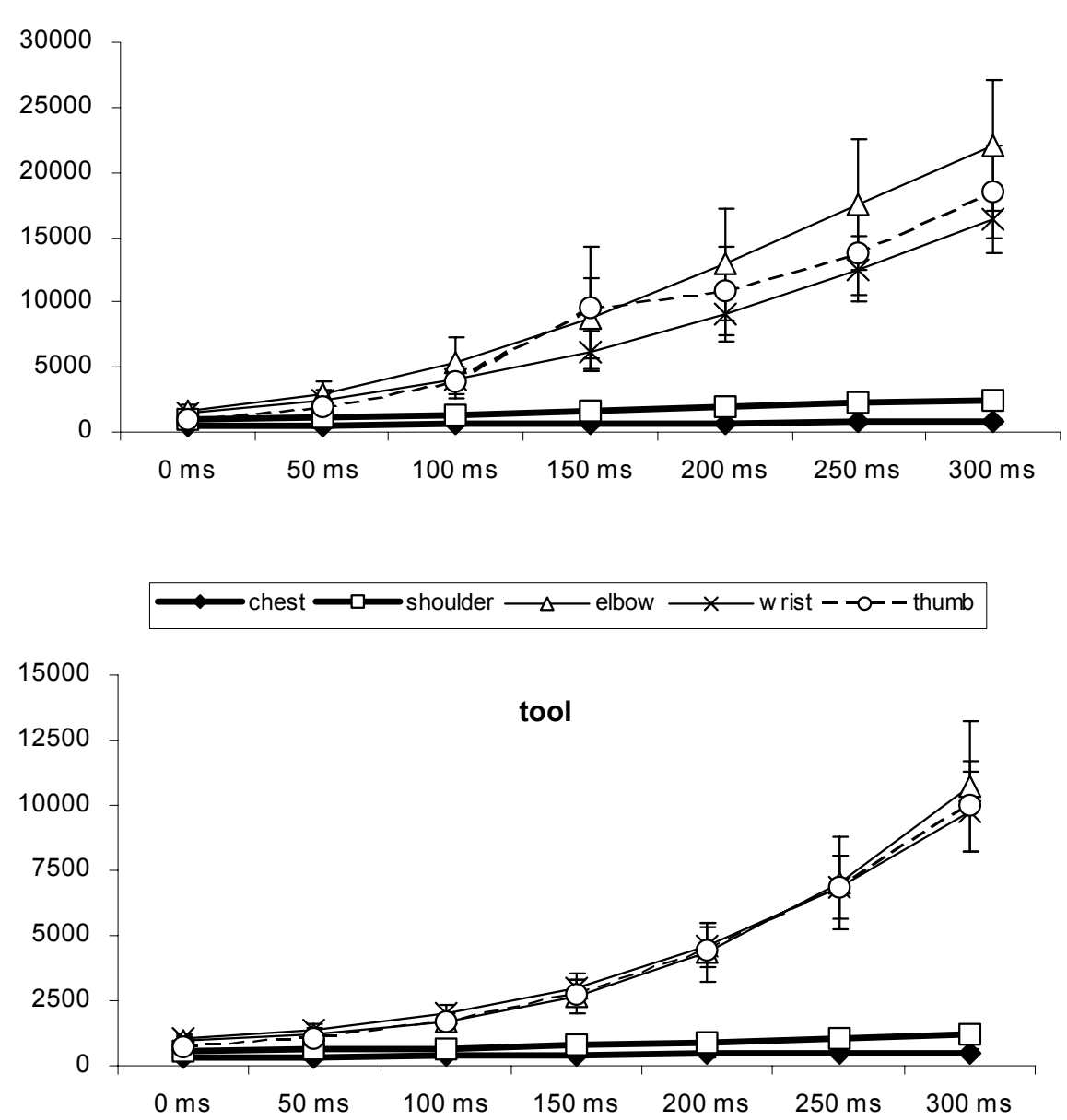

Figure 3. Path variability calculated from body sites prior to contact of the object in natural (top) and remote prehension (bottom). Y axis, ellipsoid volume (standard deviations on 3 motion dimensions). X axis, time to contact of the object. Note that the scale on the $Y$ axis displayed in the tool graph is half that of the hand graph.

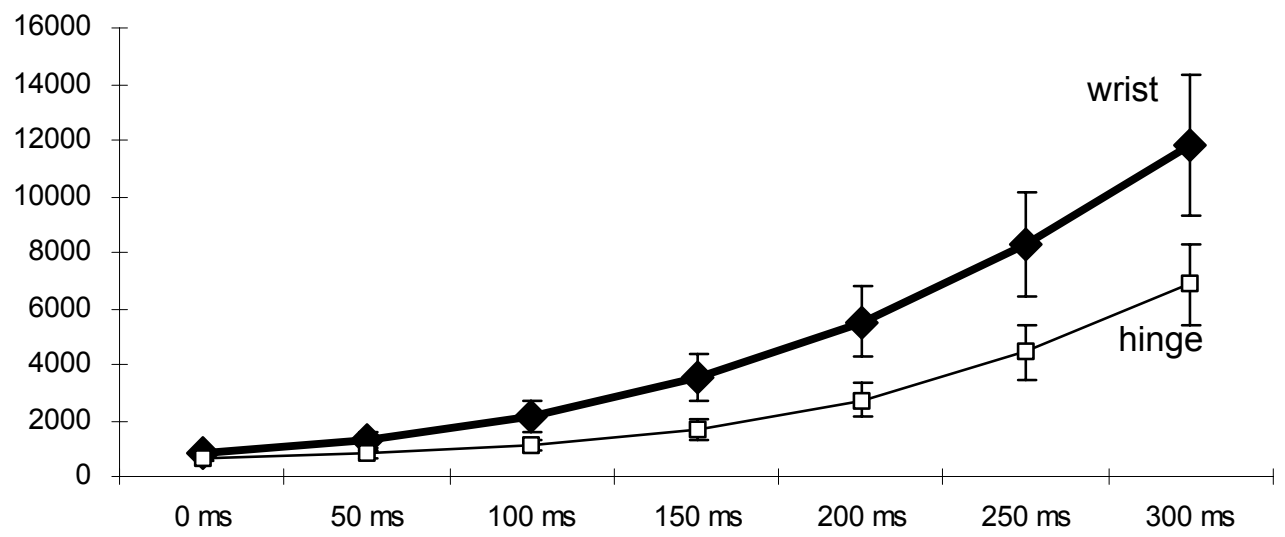

Figure 4. Path variability is smaller on the tool than the wrist. $Y$ axis, ellipsoid volume of standard deviations on 3 motion dimensions. $X$ axis, time to contact of the object. 


\section{DISCUSSION}

Generally, the results support Bernstein's notion on DOF control of human movement. Couplings between joints of the upper limbs are formed in both remote and natural prehension. However, the patterns of coupling differ between natural and remote prehension. Results suggest the formation of couplings is subject to the initial configuration of the limbs in addition to the task requirement and skill level. The coupling of joints within the limbs is modified by holding a tool in hand.

With so many variable combinations of motor segments, we are impressed by the fact that the movement paths of wrist and hinge were consistently preserved, and above all, the movement goal was nevertheless achieved precisely. As we see in the data, path variability from all movement segments is reduced when extra DOF are introduced by the tool; the reduction of variability is more marked from the distal than the proximal segments. When task requirements escalate, less variability is observed from the movement segments. This is confirmed by the reduced variability when reaching for the object placed on the narrow base compared to the wide base.

There are two different views in interpreting control mechanisms for movement involving multiple segments. The first view proposed that movement control was intrinsic at joint space (Soechting \& Lacquaniti, 1981; Soechting et al., 1986). The spatial path of the hand by this notion should curve and be subject to the movements of joints. The second view proposed that the movement control was extrinsic at hand space; joints might go through complex angular changes in order to ensure the spatial path of the hand in a straight line (Rogosky \& Rosenbaum, 2000).

If compound movements that required coordination among multiple movement segments, such as remote prehension, were controlled in joint space, different joint coupling patterns would yield significantly different hand and tool trajectories. However, if movement was controlled at hand space, varied joint couplings would be observed to serve for movement consistency at the end-effectors of the motor system. Results of this study suggest that the end-point control strategy was applied, and application is effective to both natural and remote prehension. In other words, a multiple segment movement was controlled by extrinsic coordinates in hand space of natural prehension or even further, in the tool space of remote prehension.

Studying DOF control of tools has implications for tool design and better usage of tools. It is not uncommon to see a tool which has been designed with increasing flexibility and better suited for multiple proposes. However, it is important for a designer to keep in mind that excess DOF of a tool may reduce the manipulability of the tool. Superfluous mental stresses which accompany using an ill-designed tool may lead to adverse outcomes in manipulation. Iatrogenic injures in the gastrointestinal system resulting from malpractices with a therapeutic endoscope may serve as examples for poor performances with complex tools.

In a situation where a complex tool has to be utilized, users are required to go through a valid training course to build sufficient competency for safe manipulation of the tool. One of the training goals for using a complex tool would be to help users build coordination with the tool, so that the users are able to handle DOF of the tool with confidence. When appropriately trained, Bernstein stated that a functional coordination will be constructed among movement segments. Results from this study suggest that this coordination extends to a tool in hand. Certainly the processes for building handtool coordination are varied by tool properties and task requirement. Therefore, different training curricula are demanded for different tool use situations.

\section{ACKNOWLEDGEMENTS:}

This research was supported by doctoral fellowships to B. Zheng from Simon Fraser University and Michael Smith Foundation for Health Research, and by research grants to C. L. MacKenzie by the Natural Sciences and Engineering Research Council of Canada.

\section{REFERENCES:}

Bernstein, N. A. (1967). Co-ordination and regulation of movements. Oxford: Pergamon.

Bertram, C. P. (2002). Motor control in compound movements involving prehension. Unpublished $\mathrm{Ph} \mathrm{D}$ Thesis, Simon Fraser University, Burnaby, BC, Canada.

Elliott, D., Roy, E. A., \& Goodman, D. (1993). Asymmetries in the preparation and control of manual aiming movements. Canadian Journal of Experimental Psychology, 47(3), 570-589.

Georgopoulos, A. P., Kalaska, J. F., \& Massey, J. T. (1981). Spatial trajectories and reaction times of aimed movements: Effects of practice, uncertainty, and change in target location. Journal of Neurophysiology, 46(4), 725-743.

Paulignan, Y., MacKenzie, C. L., Marteniuk, R. G., \& Jeannerod, M. (1991). Selective perturbation of visual input during prehension movements. 1 . The effects of changing object position. Experimental Brain Research, 83(3), 502-512.

Rogosky, B. J., \& Rosenbaum, D. A. (2000). Frames of reference for human perceptual-motor coordination: Space-based versus joint-based adaptation. Journal of Motor Behavior, 32(3), 297-304.

Roy, E. A., \& Elliott, D. (1986). Manual asymmetries in visually directed aiming. Canadian Journal of Psychology, 40(2), 109-121.

Soechting, J. F., \& Lacquaniti, F. (1981). Invariant characteristics of a pointing movement in man. Journal of Neuroscience, 1(7), 710-720.

Soechting, J. F., Lacquaniti, F., \& Terzuolo, C. A. (1986). Coordination of arm movements in three-dimensional space. Sensorimotor mapping during drawing movement. Neuroscience, 17(2), 295-311.

Vereijken, B., Whiting, H. T., \& Beek, W. J. (1992). A dynamical systems approach to skill acquisition. The Quarterly Journal of Experimental Psychology. A, Human Experimental Psychology, 45(2), 323-344.

Whiting, H. T. A., \& Bernstein, N. A. (1984). Human motor actions: Bernstein reassessed. Amsterdam; New York: North-Holland. 